\title{
Complementary and alternative medicine usage amongst multiple sclerosis patients in Iran
}

\author{
${ }^{1,2}$ Aliyeh Ranjbar, ${ }^{3}$ Babak Daneshfard, ${ }^{2,4}$ Sadegh Izadi \\ ${ }^{1}$ Student Research Committee, ${ }^{2}$ Clinical Neurology Research Center, Shiraz University of Medical \\ Sciences, Shiraz; ${ }^{3}$ Integrative Medicine Research Unit, Chronic Respiratory Diseases Research Center, \\ National Research Institute of Tuberculosis and Lung Diseases (NRITLD), Shahid Beheshti University \\ of Medical Sciences, Tehran; ${ }^{4}$ Department of Neurology, School of Medicine, Shiraz University of \\ Medical Sciences, Shiraz, Iran.
}

\begin{abstract}
Objectives: The aim of this study was to determine the prevalence and patterns of complementary and alternative medicine (CAM) usage amongst multiple sclerosis (MS) patients in Iran and to evaluate the perceived effectiveness. Methods: This was a descriptive cross-sectional study. Data were collected from 194 patients with MS at the neurology clinics affiliated with Shiraz University of Medical Sciences by a semi-structured questionnaire. Results: The prevalence of CAM usage was $67.9 \%$. Herbal medicine was the most common CAM used among the patients (64.2\%). Cupping therapy, relaxation therapies, acupuncture, leech therapy were the other common CAM modalities used. Although less commonly used, yoga had the highest perceived effectiveness.

Conclusions: Due to the high prevalence of CAM usage, physicians should inform their patients about the advantages and disadvantages of CAM so as to help the patients make an informed choice.
\end{abstract}

Keywords: Complementary medicine, alternative therapies, Persian medicine, integrative medicine, multiple sclerosis, neurology

\section{INTRODUCTION}

Multiple sclerosis (MS) is an autoimmune disease of the central nervous system that is associated with the destruction of myelin sheath during the inflammatory process. Currently, 2.5 million people suffer from MS worldwide. ${ }^{1}$ Studies have shown an increase in the prevalence of MS in Iran from 2011 until 2016, and Fars is one of the high-risk provinces, with the incidence rate sharply increasing in the last decade. ${ }^{2-4}$ The incidence of MS is two to three times higher in women, and the average age for its presentation is approximately 34 years. ${ }^{5}$

The etiology of MS is linked to interactions between genetic and environmental factors, such as geographical latitude, vitamin D deficiency, smoking, obesity, air pollution, and viral infections. ${ }^{6}$ MS can affect different parts of the central nervous systems to a various degree and cause a myriad of neurological symptoms and comorbidities, which might significantly reduce the quality of life in the affected individuals. ${ }^{\text {? }}$

There is no definite cure for MS. The aims of treatment include accelerating the recovery from attacks, preventing the disease progression and to control MS symptoms. ${ }^{8}$ Various drugs can be useful in relieving MS symptoms, such as amantadine, methylphenidate, and modafinil for fatigue $^{9}$; selective serotonin reuptake inhibitors are preferred choice for depression ${ }^{10}$, and tricyclic antidepressants and antiepileptic drugs for the management of pain. ${ }^{11}$

Over the last decades, the use of complementary and alternative medicine (CAM) has considerably increased amongst patients with chronic diseases, including MS. ${ }^{12} \mathrm{CAM}$ is mostly utilized to control disease symptom. The high costs, unpleasant side effects, apparent inability of conventional modalities to cure chronic diseases, and patient dissatisfaction are some of the reasons for patients turning to CAM. ${ }^{13-15}$ On the other hand, patients usually receive more personalized care and psychological support (known as 3 Ts: talk, time, and touch) from CAM practitioners. ${ }^{16}$

In Iran, traditional medicine is a core component of Iranian culture. Persian Medicine $(\mathrm{PM})$ is one of the oldest holistic medical schools

Address correspondence to: Sadegh Izadi, Department of Neurology, School of Medicine, Shiraz University of Medical Sciences, Shiraz, Iran. Tel/Fax: +987136121065, Email addresses: izadisad@sums.ac.ir

Date of Submission: 14 May 2021; Date of Acceptance: 20 August 2021

https://doi.org/10.54029/2021zfv 
which includes herbal and diet therapy, manual procedures, and mind-body interventions. ${ }^{17,18}$ It is a popular branch of CAM and its usage is common in the treatment of chronic and non-communicable diseases. ${ }^{19-22}$ However, the prevalence and patterns of CAM usage amongst patients with MS in Iran is largely unknown.

The aims of this study were to determine the prevalence of CAM usage amongst patients with MS, list specific CAM amongst them, describe sociodemographic and disease characteristics of those who used CAM, and to document the perceived effectiveness.

\section{METHODS}

\section{Population and sampling}

This is a cross-sectional descriptive study. The study population was patients with MS who were referred to the Imam Reza clinic in Shiraz between April 2018 and August 2019. A total of 194 patients was selected through convenience sampling. Inclusion criteria were age 18 years or older, confirmed MS diagnosis according to the McDonald diagnostic criteria ${ }^{23}$, one year or more elapsed time since diagnosis, ability to verbally communicate in Persian language, and willingness to participate in this study.

\section{Outcome measures}

A semi-structured questionnaire including sociodemographic variables, disease variables, and aspects of CAM usage was designed by the researchers according to the related literature. ${ }^{12,24-28}$ Face validity, the time needed to administer, clarity, and logical flow of the questionnaire was determined by the researchers and was piloted in 30 MS patients. No changes were required after the pilot study.

The questionnaire was divided into three sections: the first part was related to the patients' sociodemographic characteristics including age, gender and education. The second part covered disease related characteristics such as type of MS and duration of illness. MS type was determined based on the patients' records. The third section concerned the use of CAM, level of knowledge about CAM, history of CAM usage, type of CAM used (including acupuncture, aromatherapy, leech therapy, bee therapy, herbal medicine, massage therapy, homeopathy, yoga, biofeedback or other CAM methods mentioned by the participants.), source of information about CAM, the perceived effectiveness of CAM, their belief about the effect of foods with cold nature (such as dairy products) on MS symptoms, and the daily servings of dairy products.

\section{Statistical analysis}

Data was analyzed using SPSS software (version 21). Frequency, percentage and other descriptive statistics were used to describe and summarize the data. Chi-square tests and independent t-test were applied to examine the relationship between participants' demographics/health-related factors and use of CAM. P $<0.05$ was considered as the level of statistical significance.

\section{RESULTS}

This study included a total number of 194 MS patients. The mean age of participants was 35 years (age range 17-58, SD 7). Incidence of relapsing-remitting MS, primary-progressive MS, secondary-progressive MS , progressive-relapsing MS in participants were $95.1 \%, 3.5 \%, 2.2 \%$, and $0 \%$, respectively. Rituximab and interferon were the most commonly used medications amongst the participants. Comparison of socio-demographic and disease characteristics of CAM users and non-users showed that there were no significant differences between the two groups in terms of age, gender, and education level. Although there was no significant difference in the mean duration of the disease between CAM users and non-users, a significant number of patients converted to CAM after the early years of the disease. Demographic data and disease characteristics are shown in Table 1.

In this study, the prevalence of CAM usage was $67.9 \%(n=131)$. Ten percents of patients used CAM to control disease symptoms such as reducing fatigue and depression, and improving sleep and memory; $3.9 \%$ used it to prevent exacerbation of MS; while $85.9 \%$ used it for both purposes.

Herbal therapies were the most common type of CAM used among the patients $(64.2 \%$, $\mathrm{n}=115)$. Cupping therapy was used in $10.6 \%$ $(\mathrm{n}=19)$, yoga in $8.9 \%(\mathrm{n}=16)$, acupuncture in $7.8 \%(\mathrm{n}=14)$, leech therapy in 5\% $(\mathrm{n}=9)$, and massage therapies in $1.6 \%(n=3)$. Less than $1 \%$ of them used bee therapy, homeopathy or psymentology methods. Psymentology is a recently introduced Iranian mind-body medicine which addresses the mental and psychic bodies as separate aspects of human being and mainly deals with the mental diseases. ${ }^{29}$ The use of aromatherapy and biofeedback was not reported. 
Table 1: Demographic characteristics of the participants

\begin{tabular}{llll}
\hline Characteristic & $\begin{array}{l}\text { Users of CAM/dietary } \\
\text { interventions, } \mathbf{( \% )}\end{array}$ & $\begin{array}{l}\text { Non-users of CAM/dietary } \\
\text { interventions, } \mathbf{~ ( \% )}\end{array}$ & P-value \\
\hline Demographic factors & & & \\
\hline Sex: & $28(21.4 \%) / 103(78.6 \%)$ & $16(25.8 \%) / 46(74.2 \%)$ & 0.493 \\
$\begin{array}{l}\text { Male/Female } \\
\text { Age (Year) (Mean } \pm \text { SD) }\end{array}$ & $34.87 \pm 7.51$ & $35.31 \pm 8.7$ & 0.176 \\
Education: & $21(16 \%)$ & $14(22.6 \%)$ & \\
Under diploma & $51(38.9 \%)$ & $24(38.7 \%)$ & 0.5 \\
Diploma & $59(45 \%)$ & $24(38.7 \%)$ & \\
Academic education & & & \\
\hline Health-related factors & & & \\
\hline Duration of disease & $7 \pm 3.7$ & $7.8 \pm 5.97$ & \\
Mean \pm SD & $10(7.7 \%)$ & $16(25.8 \%)$ & \\
$<2$ years & $71(54.6 \%)$ & $21(33.9 \%)$ & \\
3-7 years & $36(27.7 \%)$ & $9(14.5 \%)$ & \\
8-12 years & $13(10 \%)$ & & \\
$>12$ years & & & \\
\hline
\end{tabular}

CAM: Complementary and Alternative Medicine; SD: Standard Deviation

Twenty nine percent of the patients used more than one type of CAM. Eleven percent of the patients perceived CAM to be highly efficacious, $42.6 \%$ of them perceived it to be of moderate efficacy, $37.2 \%$ perceived that it had little effect, and $9.3 \%$ reported that it was ineffective. The dissatisfaction with CAM resulted in $11 \%$ of patients quitting it. The highest perceived efficacy was related to yoga (average of scores: 3.2 , refer to
Table 2 for scale) while acupuncture had the lowest perceived efficacy (average of scores: 1.7). Data of CAM used and perception of CAM benefit are summarized in Table 2.

In addition, the results showed that perceived efficacy was higher in patients with more information about CAM $(\mathrm{P}<0.05)$. However, age, sex, and duration of the disease was not related to perceived efficacy $(\mathrm{P}=0.21, \mathrm{P}=0.13, \mathrm{P}=0.75)$.

Table 2: Types of CAM used and perception of CAM benefit

\begin{tabular}{|c|c|c|c|c|c|c|}
\hline \multirow[b]{2}{*}{ Types of CAM } & \multirow[b]{2}{*}{$\begin{array}{c}\text { Total use } \\
\text { n (\%) }\end{array}$} & \multicolumn{5}{|c|}{ Perception of CAM Benefit } \\
\hline & & $\begin{array}{c}\text { Very } \\
\text { effective } \\
\text { (score 4) } \\
\text { n }(\%)\end{array}$ & $\begin{array}{c}\text { Moderate } \\
\text { effective } \\
\text { (score 3) } \\
\text { n }(\%)\end{array}$ & $\begin{array}{c}\text { Low effective } \\
\text { (score 2) } \\
\text { n }(\%)\end{array}$ & $\begin{array}{c}\text { Ineffective } \\
\text { (score 1) } \\
\text { n }(\%)\end{array}$ & $\begin{array}{l}\text { Mean } \\
\text { of the } \\
\text { score }\end{array}$ \\
\hline Herbal therapy & $115(64.2 \%)$ & $11(9.6 \%)$ & $44(38.2 \%)$ & $49(42.6 \%)$ & $11(9.6 \%)$ & 2.5 \\
\hline Cupping therapy & $19(10.6 \%)$ & $1(5.2 \%)$ & $6(31.6 \%)$ & $10(52.6 \%)$ & $2(10.5 \%)$ & 2.3 \\
\hline Yoga & $16(8.9 \%)$ & $6(37.5 \%)$ & $6(37.5 \%)$ & $3(18.8 \%)$ & $1(6.2 \%)$ & 3.1 \\
\hline Acupuncture & $14(7.8 \%)$ & $1(7.1 \%)$ & $5(35.7 \%)$ & $8(57.1)$ & 0 & 2.5 \\
\hline Leech therapy & $9(5 \%)$ & $1(11.1 \%)$ & $4(44.4 \%)$ & $4(44.4 \%)$ & 0 & 2.6 \\
\hline Massage therapy & $3(1.6 \%)$ & 0 & $2(66.6 \%)$ & $1(33.3 \%)$ & 0 & 2.3 \\
\hline Bee therapy & $1(.5 \%)$ & 0 & 0 & 1 & 0 & 2 \\
\hline Homeopathy & $1(.5 \%)$ & 0 & 1 & 0 & 0 & 3 \\
\hline Psymentology method & $1(.5 \%)$ & 0 & 0 & 1 & 0 & 2 \\
\hline
\end{tabular}

CAM: Complementary and Alternative Medicine. 
Patients' estimation of their information about the use of CAM in the treatment of MS showed that $10.9 \%$ of them thought that they had a lot of information, $29.5 \%$ moderate information, $39.9 \%$ low, and $19.7 \%$ none. The source of their information was a family member or friend in $42.3 \%$ of the cases, $30.4 \%$ from social media or internet reports, $13.6 \%$ from TV programs, $9 \%$ from other MS patients, and $4.5 \%$ from health professionals. The use of CAM in $4.8 \%$ of patients resulted in irregular use of medication. $4 \%$ of patients stopped their MS medication after initiating CAM. The median duration of medication use was 7 months. Ninety percent of them continued their conventional treatment after starting CAM. Seventy percent of patients had the tendency to continue using the current CAM. Forty two percent of them were interested in using other types of CAM and $61.5 \%$ of patients recommended CAM to other patients.

In total, $81.7 \%$ of patients believed that foods of a "cold" nature such as dairy products exacerbate the symptoms of MS. The daily serving of dairy foods was reportedly to be less than one serving in $8.8 \%$ of patients, one serving in $43 \%$, two servings in $40.9 \%$, and three or more servings in $7.7 \%$.

\section{DISCUSSION}

The result of this study showed that more than half of MS patients $(67.9 \%)$ in Shiraz, Iran used CAM which was similar to the result of previous studies in the USA, Germany, Australia, and Turkey in the last two decades. ${ }^{12,24,25,27,30}$ In the current study, there were no statistically significant differences in age, gender, type of MS, and level of education in those using CAM. The difference in duration of MS among CAM user and non-user was significant. The prevalence of CAM usage was low in MS patients in the first two years of disease, which was similar to the previous studies..$^{24,30,25}$ However, according to the literature, the high rate of CAM usage was associated with being middle-aged and having high income, high education level, long duration of diseases, and female gender. ${ }^{24,30,31}$ This conflicting finding might be due to the differences in the beliefs, values, and cultural features of the communities studied, in addition to the differences of public health systems.

Our findings showed that herbal therapy, cupping therapy, yoga, acupuncture, and leech therapy were the five most common used CAM modalities. The most common CAM methods used in the USA have been herbal therapy, chiropractic, and massage therapy ${ }^{24,27}$ while it has been herbal therapy and relaxation therapies in Eastern Turkey $^{30}$, and exercise therapy in Germany. ${ }^{25}$ The use of a particular CAM is thus associated with the geographical area and culture. In this study, herbal therapy was the most common used CAM which is similar to the study by Nayak et al. in the USA with the rate of $27 \%{ }^{24}$, and Dayapoglu and Tan in Eastern Turkey with the rate of $55.5 \% .^{30}$ However, the rate of herbal therapy in this study (64.2\%) was significantly higher compared to similar studies. The high rate of herbal therapy might be due to the frequent use of herbs in PM. Other reasons could be the rich variety of herbs and easy access to them in Iran in addition to the general belief in the safety of herbs. However, this belief could result in inappropriate consumption of medicinal herbs and consequently, adverse effects. ${ }^{32}$

Cupping therapy was the second most common CAM method whereas its usage was not report in any previous studies amongst MS patient. This paradox can be explained by the fact that Iranian people use cupping therapy as part of their religious beliefs. ${ }^{33}$

Yoga has had the highest perceived efficacy. In similar studies, highest perceived efficacy was given to relaxation and mind-body therapies such as prayer, exercise, yoga, relaxation techniques, and massage..$^{30,24}$ The effect of relaxation and mind-body therapies may have been simply at the psychological or emotional levels by reducing stress, a known factor that exacerbates MS symptoms. Various studies suggested the positive impact of yoga on reducing MS related symptoms such as depression, pain, fatigue, and neurogenic bladder dysfunction in addition to the improvement in quality of life and strength. ${ }^{34-36}$ Despite high perceived efficacy and safety of relaxation and mind-body therapies, there was lower use of yoga in this study. This could be attributed to the fact that some of the therapies that were perceived as effective may not be easily available or affordable; hence, are not frequently used.

More than half of the patients in this study did not have adequate information about CAM. Family members or friends and social media or the internet were the most frequent used sources like the studies by Leong et al. ${ }^{12}$ and Dayapoglu and Tan. ${ }^{30}$ Physician and health professionals were the source of information in only $4.5 \%$ of the cases. This finding is in line with similar studies elsewhere. ${ }^{37,38}$ In Nayak's study, physicians 
recommended CAM treatment in $15.3 \%$ of patients. ${ }^{24}$

Herbal medicines without suitable quality control and adequate labeling are easily available in the market. ${ }^{39}$ The general population considers herbal medicines as very safe without any adverse effect. However, studies have shown that inappropriate use of herbal medicines could be associated with hepatotoxicity, nephrotoxicity, cardiotoxicity, neurotoxicity, and allergic reactions. ${ }^{32}$ Herbal supplements might also adversely interact with the commonly used drugs in MS. ${ }^{40,41}$

On the other hand, the traditional beliefs and culture of Iranian people have resulted in a large percentage of patients to limit the consumption of "cold-natured" foods such as dairy products. It should be mentioned that PM is based on the theory of Mizaj (temperament) and humors (i.e., sanguine, phlegm, black bile, and yellow bile) including the quadruple qualities of hotness, coldness, wetness, and dryness).$^{20}$ Any imbalance among these qualities (also called dystemperament) may result in a disease. With regards to MS, it was believed that their general Mizaj tended toward "coldness". ${ }^{42}$

Furthermore, the use of CAM in some of the patients led to cessation or irregular usage of their conventional treatments. These challenges coupled with the high prevalence of CAM usage and the willingness of patients to continue using CAM show the necessity to ask patients about CAM usage during their routine visits. Physicians should discuss the type of CAM therapies and their advantages and disadvantages to help their patients to make informed and safe choices.

There were several limitations in this study. First, the small sample led to difficulties in statistical interpretation of the results. Second, this sample reflects only one region of Iran; hence, the results cannot be generalized to all MS patients in Iran. Therefore, future studies should include larger samples from different regions. Moreover, the sample was selected from patients who had been referred to neurology clinics and were likely to have excluded patients who only used unconventional therapy. However, previous studies showed very few patients who only use unconventional therapies. Another limitation was the use of self-reporting in evaluating the efficacy and hence, did not include clinical evidence of efficacy.

This study provides a general overview of the pattern of CAM usage but further studies are warranted to explore the details of CAM usage, in particular herbal therapy and the type of herbal remedies, and their possible adverse effects in MS patients. The accurate evaluation of the CAM efficacy in the management of distinct MS symptoms and prevention of exacerbation are other issues that should be considered in the future studies. In addition, future studies should determine the physicians and health professionals' level of information about CAM. Moreover, the evaluation of the efficacy of yoga or similar therapy on the impact on mood disorder in MS, given the high level of perceived efficacy of yoga on psychological effect, and the high prevalence of depression and anxiety among MS patients is an interesting topic to be explored in future studies.

In conclusion, many patients with MS in Iran use CAM therapies. The use of these interventions was higher amongst patients after first two years of illness. Herbal medicine is the most common CAM therapy among patients with MS. Yoga is the most effective CAM therapy according to patient's perceptions. Family members, friends, social media and the internet were more significant sources of information about CAM. Restrictions on the use of certain essential foods including dairy products and a reduction in their adherence to the conventional treatment of MS are some of the challenges that CAM brought about. Given the high prevalence of CAM usage amongst MS patients, physicians should improve their knowledge on CAM and routinely ask their patients about CAM utilization so as to advise them on the proper use of CAM.

\section{ACKNOWLEDGEMENTS}

The authors wish to thank Mr. H. Argasi at the Research Consultation Center (RCC) of Shiraz University of Medical Sciences for his invaluable assistance in editing this manuscript.

\section{DISCLOSURE}

Financial support: This study approved and financially supported by the Shiraz University of Medical Sciences.

Conflicts of interest: None

\section{REFERENCES}

1. Briggs FB, Hill E. Estimating the prevalence of multiple sclerosis using 56.6 million electronic health records from the United States. Mult Scler 2020; 26(14):1948-52.

2. Izadi S, Nikseresht AR, Poursadeghfard M, 
Borhanihaghighi A, Heydari ST. Prevalence and incidence of multiple sclerosis in Fars province, Southern Iran. Iran J Med Sci 2015;40(5):390.

3. Izadi S, Nikseresht A, Poursadeghfard M. Epidemiology of multiple sclerosis in Fars province. Iran J of Epidemiol 2014;10(2):56-61.

4. Azami M,YektaKooshali MH, Shohani M, Khorshidi A, Mahmudi L. Epidemiology of multiple sclerosis in Iran: A systematic review and meta-analysis. PloS One 2019;14(4):e0214738.

5. Ascherio A, Munger KL, Lunemann JD. The initiation and prevention of multiple sclerosis. Nat Rev Neurol 2012;8(11):602-12.

6. Khan F, McPhail T, Brand C, Turner-Stokes L, Kilpatrick T. Multiple sclerosis: disability profile and quality of life in an Australian community cohort. Int J Rehabil Res 2006;29(2):87-96.

7. de Sa JCC,Airas L, Bartholome E, et al. Symptomatic therapy in multiple sclerosis: a review for a multimodal approach in clinical practice. Ther $A d v$ Neurol Disord 2011;4(3):139-68.

8. Salvetti M, Landsman D, Schwarz-Lam P, Comi G, Thompson AJ, Fox RJ. Progressive MS: from pathophysiology to drug discovery. Mult Scler 2015;21(11):1376-84.

9. Nourbakhsh B, Revirajan N, Waubant E. Treatment of fatigue with methylphenidate, modafinil and amantadine in multiple sclerosis (TRIUMPHANTMS): Study design for a pragmatic, randomized, double-blind, crossover clinical trial. Contemp Clin Trials 2018;64:67-76.

10. Hind D, Cotter J, Thake A, et al. Cognitive behavioural therapy for the treatment of depression in people with multiple sclerosis: a systematic review and meta-analysis. BMC Psychiatr 2014;14(1):5 .

11. Poellmann W, Feneberg W. Current management of pain associated with multiple sclerosis. CNS Drugs 2008;22(4):291-324.

12. Leong EM, Semple S, Angley M, Siebert W, Petkov J, McKinnon R. Complementary and alternative medicines and dietary interventions in multiple sclerosis: what is being used in South Australia and why? Complement Ther Med 2009;17(4):216-23.

13. Shakeri A, Hashempur MH, Mojibian M, Aliasl F, Bioos S, Nejatbakhsh F. A comparative study of ranitidine and quince (Cydonia oblonga mill) sauce on gastroesophageal reflux disease (GERD) in pregnancy: a randomised, open-label, active-controlled clinical trial. J Obstet Gynaecol 2018;38(7):899-905

14. Khiveh A, Hashempur MH, Shakiba M, et al. Effects of rhubarb (Rheum ribes L.) syrup on dysenteric diarrhea in children: a randomized, double-blind, placebo-controlled trial. J Integr Med 2017;15(5):365-72.

15. Akrami R, Hashempur MH, Tavakoli A, et al. Effects of Fumaria parviflora L on uremic pruritus in hemodialysis patients: a randomized, double-blind, placebo-controlled trial. Jundishapur J Natural Pharmaceutical Products 2016;12(3).

16. Daneshfard B, Sanaye MR, Nimrouzi M.Prolegomena to a true integrative medical paradigm. Alternative Ther Health Med 2019;25(2):50-60
17. Atarzadeh F, Daneshfard B, Dastgheib L, Jaladat AM, Amin G. Early description of diet-induced blistering skin diseases in medieval Persia: Avicenna's point of view. Skinmed 2016;14(5):367-70.

18. Golshani SA, Daneshfard B, Mosleh G, Salehi A. Drugs and pharmacology in the Islamic middle wra. Pharm Hist (Lond) 2015;45(3):64-9.

19. Daneshfard B, Yekta NH, Khoshdel A, Heiran A, Cheraghi R, Yarmohammadi H. The effect of Delphinium denudatum (Jadwar) on fatigue: A randomized double blind placebo-controlled clinical trial. Complement Ther Med 2019;46:29-35.

20. Nimrouzi M, Daneshfard B, Tafazoli V, Akrami R. Insomnia in traditional Persian medicine. Acta Medico-Historica Adriatica: AMHA 2019;17(1):4554.

21. Nimrouzi M, Tafazoli V, Daneshfard B, Zare M. Optimal fluid intake in daily diet: Avicenna's view. J Integr Med 2016;14(4):241-4.

22. Hashempur MH, Ghasemi MS, Daneshfard B, et al. Efficacy of topical chamomile oil for mild and moderate carpal tunnel syndrome: A randomized double-blind placebo-controlled clinical trial. Complement Ther Clin Pract 2017;26:61-7.

23. McDonald WI, Compston A, Edan G, et al. Recommended diagnostic criteria for multiple sclerosis: guidelines from the International Panel on the diagnosis of multiple sclerosis. Ann Neurol 2001;50(1):121-7.

24. Nayak S, Matheis RJ, Schoenberger NE, Shiflett SC. Use of unconventional therapies by individuals with multiple sclerosis. Clin Rehabil 2003;17(2):181-91.

25. Apel A, Greim B, Konig N, Zettl UK. Frequency of current utilisation of complementary and alternative medicine by patients with multiple sclerosis. J Neurol 2006;253(10):1331-6.

26. Pucci E, Cartechini E, Taus C, Giuliani G. Why physicians need to look more closely at the use of complementary and alternative medicine by multiple sclerosis patients. Eur J Neurol 2004;11(4):263-7.

27. Marrie RA, Hadjimichael O, Vollmer T. Predictors of alternative medicine use by multiple sclerosis patients. Mult Scler 2003;9(5):461-6.

28. Shinto L, Yadav V, Morris C, Lapidus J, Senders A, Bourdette D. The perceived benefit and satisfaction from conventional and complementary and alternative medicine (CAM) in people with multiple sclerosis. Complement Ther Med 2005;13(4):264-72.

29. Taheri MA, Bayyazi A. Definition of psymentology, an Iranian complementary and alternative medicine. Procedia-Social and Behavioral Sci 2013;84:153449.

30. Dayapoglu N, Tan M. Use of complementary and alternative medicine among people with multiple sclerosis in Eastern Turkey. Neurol Asia 2016;21(1):63-71.

31. MacLennan AH, Myers SP, Taylor AW. The continuing use of complementary and alternative medicine in South Australia: costs and beliefs in 2004. Med J Australia 2006;184(1):27-31.

32. Pasalar M, Daneshfard B, Lankarani KB. Complementary and alternative medicine-related drug-induced liver injury in Iran. J Clin Transl Hepatol 2020;8(1):106. 
33. Nimrouzi M, Mahbodi A, Jaladat AM, Sadeghfard A, Zarshenas MM. Hijamat in traditional Persian medicine: risks and benefits. J Evid Based Complementary Altern Med 2014;19(2):128-36.

34. Frank R, Larimore J. Yoga as a method of symptom management in multiple sclerosis. Front Neurosci 2015;9:133.

35. Rogers KA, MacDonald M. Therapeutic yoga: symptom management for multiple sclerosis. J Altern Complement Med 2015;21(11):655-9.

36. Senders A, Wahbeh H, Spain R, Shinto L. Mind-body medicine for multiple sclerosis: a systematic review. Autoimmune Dis 2012;2012:567324.

37. Giveon SM, Liberman N, Klang S, Kahan E. Are people who use "natural drugs" aware of their potentially harmful side effects and reporting to family physician? Patient Educ Couns 2004;53(1):511.

38. Stuifbergen AK, Harrison TC. Complementary and alternative therapy use in persons with multiple sclerosis. Rehabil Nurs 2003;28(5):141-7.

39. Raynor DK, Dickinson R, Knapp P, Long AF, Nicolson DJ. Buyer beware? Does the information provided with herbal products available over the counter enable safe use? BMC Med 2011;9(1):94.

40. Davis SA, Feldman SR, Taylor SL. Use of St. John's wort in potentially dangerous combinations. $J$ Altern Complement Med 2014;20(7):578-9.

41. Liu R, Li X, Huang N, Fan MY, Sun R, et al. Toxicity of traditional Chinese medicine herbal and mineral products. Adv Pharmacol 2020;87:301-46.

42. Abbasian R, Mojahedi M, Alizadeh M, et al. Mizaj assessment in multiple sclerosis (MS) patients based on Persian medicine. J Complement Integr Med 2021. Doi: $10.1515 /$ jcim-2020-0428. 